\title{
Association between cooking oil fume exposure and lung cancer among Chinese nonsmoking women: a meta-analysis
}

This article was published in the following Dove Press journal:

OncoTargets and Therapy

19 May 2016

Number of times this article has been viewed

\section{Yingbo Xue \\ Ying Jiang \\ Shan Jin \\ Yong $\mathrm{Li}$}

Department of Oncology, Guizhou Provincial People's Hospital, Guiyang, Guizhou, People's Republic of China

Correspondence: Yingbo Xue Department of Oncology, Guizhou People's Hospital, No 83 Zhongshan East Road, Guiyang, Guizhou 550002, People's Republic of China Tel +8685। 8560 I796 Email xueyingbo0619@।26.com
Abstract: Lung cancer has been the main cause of cancer death around the world. Cigarette smoking has been identified as a risk factor for lung cancer in males. However, the etiological factors in nonsmoking women remain elusive. A meta-analysis was conducted to evaluate the relationship between cooking oil fume exposure and lung cancer among Chinese nonsmoking women. Thirteen articles containing three population-based case-control and ten hospital-based case-control studies were included in this meta-analysis. These studies with a total of 3,596 lung cancer women and 6,082 healthy controls were analyzed by RevMan 5.3. Fixed effects model or random effects model was used to obtain pooled estimates of risk ratio. The risk ratios with a $95 \% \mathrm{CI}$ were $1.74(95 \% \mathrm{CI}=1.57-1.94)$ and $2.11(95 \% \mathrm{CI}=1.54-2.89)$, respectively. Cooking oil fume exposure as well as not using a kitchen ventilator when cooking was significantly associated with lung cancer among nonsmoking women $(Z=10.07, P<0.00001 ; Z=4.65$, $P<0.00001)$. Cooking oil fume exposure, especially lacking a fume extractor, may increase the risk of lung cancer among Chinese nonsmoking women.

Keywords: cooking oil fume exposure, lung cancer, meta-analysis, nonsmoking women

\section{Introduction}

Lung cancer accounts for $\sim 17 \%$ of cancer-related deaths around the world, and cigarette smoking is regarded as the principal risk factor for lung cancer in males. ${ }^{1}$ As it is well known, other risk factors also increase the risk of lung cancer in female nonsmokers. A study demonstrated that $15 \%$ of male patients and $53 \%$ of female patients with lung cancer in five continents were not due to smoking. ${ }^{2}$ A number of epidemiological studies suggested that in lifetime never smokers, lung cancer morbidity in women was significantly higher than that in men. ${ }^{3}$ Prominent epidemiological, clinical, and pathological differences in various types of lung cancers have been discovered between lifetime never smokers and heavy smokers. ${ }^{4}$ In recent years, indoor PM2.5, ${ }^{5}$ housing characteristics, ${ }^{6}$ home passive smoking exposure,${ }^{7}$ indoor air pollution, ${ }^{8}$ cooking oil fume exposures, ${ }^{9}$ and previous respiratory disease have been demonstrated as the causes of lung cancer. ${ }^{10}$ On the other hand, numerous studies have showed that daily intake of fruits and vegetables is associated with low risk of several cancers, especially of the digestive tract. ${ }^{11}$ However, the various risk factors and complicated epidemiological feature of lung cancer in nonsmokers are not well understood.

Exposure to environmental risk factors is worthy of attention in the development of lung cancer. Lung cancer susceptibilities of population differ in the same environment due to genetic susceptibility. ${ }^{12}$ MicroRNAs play an important role in some 
biological processes, such as differentiation, apoptosis, proliferation, and progression of multiple types of diseases, including lung cancer. ${ }^{13}$ Exposure to polycyclic aromatic hydrocarbons, a harmful compound in cooking oil fumes, will lead to oxidative DNA damage and lipid peroxidation. ${ }^{14}$ Cooking oil fume exposure increased oxidative stress and endoplasmic reticulum stress, which induced cytotoxicity and apoptosis in primary fetal alveolar epithelial cells (AEC) II cells. ${ }^{15}$ Several studies have demonstrated that mutagens and carcinogens are released by fumes from heated cooking oils, using the Ames assay or SOS chromotest. ${ }^{16}$ Biology experiments in human lung adenocarcinoma cells found that various mutagenic compounds were generated by cooking oil fume that lead to DNA damage and oxidative damage to cells. ${ }^{17}$ Moreover, epidemiological investigation in Chinese nonsmoking women showed that exposure to cooking oil fume might increase the risk of lung cancer. ${ }^{18}$ Cooking oil fume exposure was significantly associated with risk of lung cancer, particularly when there is a lack of a fume extractor or ventilator. ${ }^{19}$ A previous study has shown that household stove improvement reduces the risk of lung cancer in Xuanwei, People's Republic of China. ${ }^{20}$ The Chinese-style cooking is thought to be the main cause increasing the risk of lung cancer among nonsmoking women.

Although several epidemiological studies have proved the association between cooking oil fume exposure and lung cancer, due to limitations, such as relatively small sample size and regional differences, the results are still inconclusive. Here, we, for the first time, carried out a metaanalysis of the published studies reporting the association of cooking oil fume exposure with lung cancer in Chinese nonsmoking women. We also investigated the relationship of lacking a ventilator in kitchen with risk of lung cancer in female nonsmokers.

\section{Materials and methods}

\section{Search strategy and selection criteria}

We reviewed all the available publications that reported the association of cooking oil fume exposure with lung cancer in Chinese nonsmoking women. The electronic databases, including PubMed, Embase, Web of Science, MEDLINE, and CNKI, were searched for all articles published before June 14, 2015, using the keywords "lung cancer" or "lung neoplasm" or "lung carcinoma" or "lung tumor" or "cancer" and "cooking oil fume." All studies included in this metaanalysis must meet the following criteria: 1) original study; 2) all cases were confirmed by X-ray computed tomography or histopathology; 3 ) the size of the samples and the number of patients with lung cancer with or without cooking oil fume exposure were available; 4) when the same population exists in more than one publication, we included the studies with a larger sample size; and 5) the meta-analysis was confined to English-language and Chinese-language articles. Letters to the editor, abstract, reviews, and articles published in books were excluded. All included studies were assessed by two reviewers. The Ethics Committee of Guizhou Provincial People's Hospital did not require ethics approval and patient informed consent for meta-analysis study.

\section{Data extraction}

Information of included studies was extracted by two coauthors independently using the same selection criteria, and uncertainties were analyzed by all coauthors to achieve consensus. The following data were obtained: first author, publishing year, study type, study location, study period, use of kitchen ventilator, and smoking history.

\section{Statistical analysis}

The meta-analysis of these dichotomous outcomes was conducted using Excel (Microsoft Corporation, Redmond, WA, USA) and ReviMan (computer program) Version 5.3 (The Nordic Cochrane Centre, The Cochrane Collaboration, 2014, Copenhagen, Denmark). Odds ratio (OR) with 95\% CI was used to evaluate the association between cooking oil fume exposure and lung cancer in Chinese nonsmoking women. We assessed between-study heterogeneity by $I^{2}$ statistics. $I^{2}>50 \%$ or $P$-value of $<0.05$ was considered significantly heterogeneous. Random effects model (DerSimonian-Laird) was used to estimate the pooled ORs when significant heterogeneity existed among the included studies. The pooled effect size of studies and the significant $P$-value of overall ORs were determined using a $Z$-test. A $Z$-score, a standard score, indicates how many SDs are from the mean. A $Z$-score is calculated from the following formula: $Z=(X-\mu) / \sigma$, where $Z$ is the $Z$-score, $X$ is the value of the element, $\mu$ is the mean of the population, and $\sigma$ is the SD. A funnel plot was obtained to test the publication bias.

\section{Results}

\section{Study selection}

According to the strict criteria for inclusion, 236 potentially relevant studies were identified by screening titles and abstracts, of which 194 articles were excluded for the following reasons: animal or cell experiments, no original information, letters, reviews, or abstracts. After reading the full text, four articles were excluded for overlapping information. Twenty-five 


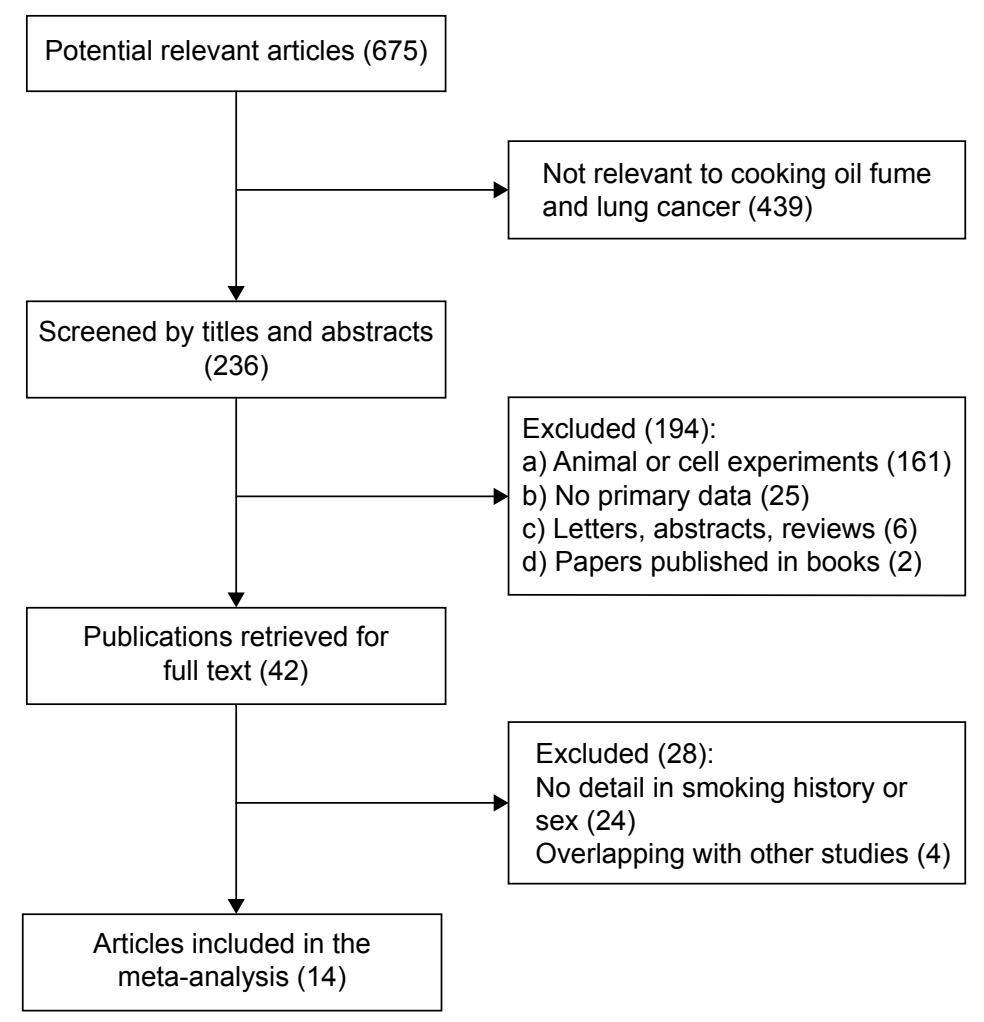

Figure I Flowchart of study identification.

articles were abandoned for lack of smoking history or sex information. Finally, 14 articles met the selection criteria and were included in this meta-analysis, as shown in Figure 1.

\section{Study characteristics}

The main characteristics of 14 case-control studies published between 1996 and 2014 are presented in Tables 1 and 2. Ten were hospital-based case-control studies, and the remaining four were population-based case-control studies. There were totally 3,596 lung cancer women and 6,082 healthy controls in the meta-analysis study sample sizes ranged from 187 to 1,603 with a mean of 745 . All of them were lifetime nonsmokers.

\section{Results of meta-analysis}

As shown in Figure 2A, the pooled estimate of the OR was acquired using the fixed effects model on account of heterogeneity across studies $\left(P=0.16 ; I^{2}=31 \%\right)$. The combined results of ten studies $(2,641$ cases and 4,076 controls) showed the association between cooking oil fume exposure and lung cancer in nonsmoking women $(\mathrm{OR}=1.74$; $95 \% \mathrm{CI}=1.57-1.94$; ten studies). Cooking oil fume exposure significantly increases the risk of lung cancer among Chinese nonsmoking women.

The meta-analysis of four studies (955 cases and 2,006 controls) demonstrated that cooking oil fume exposure with no

Table I Main characteristics of ten studies included in the meta-analysis of lung cancer associated with cooking oil fume exposure among nonsmoking women

\begin{tabular}{|c|c|c|c|c|c|c|}
\hline Study (year) & Study location & Study period & Study design & Cases (N) & Controls (N) & Language \\
\hline $\mathrm{Ko}^{27}(1997)$ & Greater Kaohsiung & $1992-1993$ & Hospital-based case-control & 310 & 306 & English \\
\hline Li et $\mathrm{al}^{21}(2008)$ & Liaoning & $2002-2006$ & Hospital-based case-control & 225 & 475 & English \\
\hline $\mathrm{Liu}^{28}(2000)$ & Shanghai & $1995-1997$ & Population-based case-control & 94 & 93 & Chinese \\
\hline $\operatorname{Liu}^{29}(200 \mathrm{I})$ & Shanghai & $1992-1993$ & Population-based case-control & 498 & 595 & Chinese \\
\hline Metayer $^{30}$ (2002) & Gansu & $1994-1998$ & Population-based case-control & 227 & 449 & Chinese \\
\hline $\mathrm{Su}^{31}(20 \mid 4)$ & Liaoning & 2004-2009 & Hospital-based case-control & 363 & 370 & Chinese \\
\hline Wang ${ }^{32}(1996)$ & Shenyang & $199 \mid-1994$ & Hospital-based case-control & 166 & 166 & Chinese \\
\hline Yin et $\mathrm{al}^{26}(2014)$ & Shenyang & $2004-2010$ & Hospital-based case-control & 306 & 318 & English \\
\hline Zhong $^{33}$ (1999) & Shanghai & $1992-1994$ & Hospital-based case-control & 504 & 601 & English \\
\hline Zhu $^{34}(20 \mathrm{lo})$ & Changsha & 2009 & Hospital-based case-control & 160 & 160 & Chinese \\
\hline
\end{tabular}

Abbreviation: $\mathrm{N}$, number. 
Table 2 Main characteristics of four studies included in the meta-analysis of lung cancer associated with use of a kitchen ventilator when cooking among nonsmoking women

\begin{tabular}{lllllll}
\hline Study (year) & Study location & Study period & Study design & Cases (N) & Controls (N) & Language \\
\hline Ko $(1997)$ & Greater Kaohsiung & $1992-1993$ & Hospital-based case-control & 310 & 306 & English \\
$\mathrm{Ko}^{35}(2000)$ & Greater Kaohsiung & $1993-1996$ & Hospital-based case-control & 339 & I,264 & English \\
Mu et al $^{8}(2013)$ & Taiyuan & $2005-2007$ & Hospital-based case-control & 153 & 216 & English \\
$\mathrm{Yu}^{36}(2006)$ & Hong Kong & $2002-2004$ & Hospital-based case-control & $25 \mathrm{I}$ & $\mathrm{I}, 069$ & English \\
\hline
\end{tabular}

Abbreviation: $\mathrm{N}$, number.

ventilator in kitchen increased the risk of lung cancer among Chinese nonsmoking women $(\mathrm{OR}=2.11 ; 95 \% \mathrm{CI}=1.54-2.89$; four studies) (Figure 2B). The pooled ORs for the risk of lung cancer were calculated with the random effects model because statistically significant heterogeneity was observed ( $\left.P=0.08 ; I^{2}=55 \%\right)$. More clinical studies should be carried out taking into account age, habit of cooking, passive smoking, histological grades, and the types of lung cancer.

\section{Publication bias and sensitivity analysis}

The publication bias of the studies included in this metaanalysis was assessed by funnel plots. The funnel plot was mainly symmetrical, suggesting that no obvious publication bias existed in this study (Figure 3). On the other hand, sensitivity analysis was performed to assess whether individual study affected the final summary results. The sensitivity analysis showed that none of the studies remarkably affected the pooled ORs and CIs, and deletion of any one study had no significant effect on the final results (data not shown).

\section{Discussion}

Cooking oil fume contains $>200$ kinds of harmful gases. Exposure to cooking oil fumes is related to the high mortality rate of lung cancer among Chinese women. ${ }^{21}$ Cooking oil fumes are mainly composed of two types of chemical compounds, including polycyclic aromatic hydrocarbons and aldehydes. ${ }^{16}$ Exposure to 2,4-decadienal has been proved to aggravate the genotoxicity of cooking oil fumes and to promote cell proliferation and cytokine levels in a human bronchial epithelial cell line. ${ }^{22}$ A study showed that DNA adducts were generated in human lung adenocarcinoma CL-3 cells when they were exposed to cooking oil fumes. ${ }^{23}$

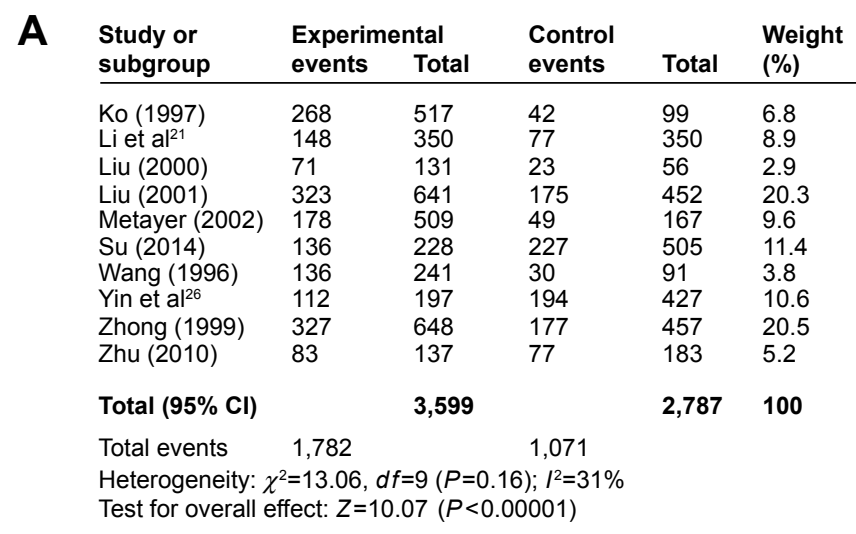

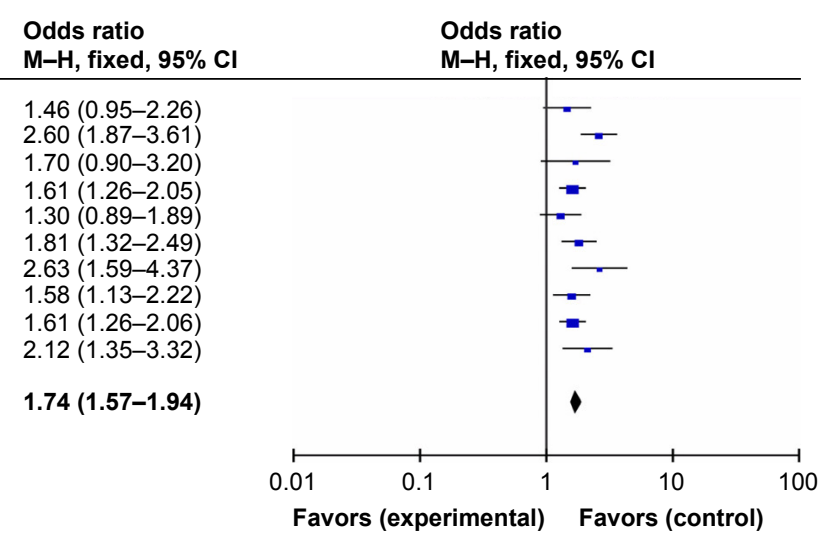

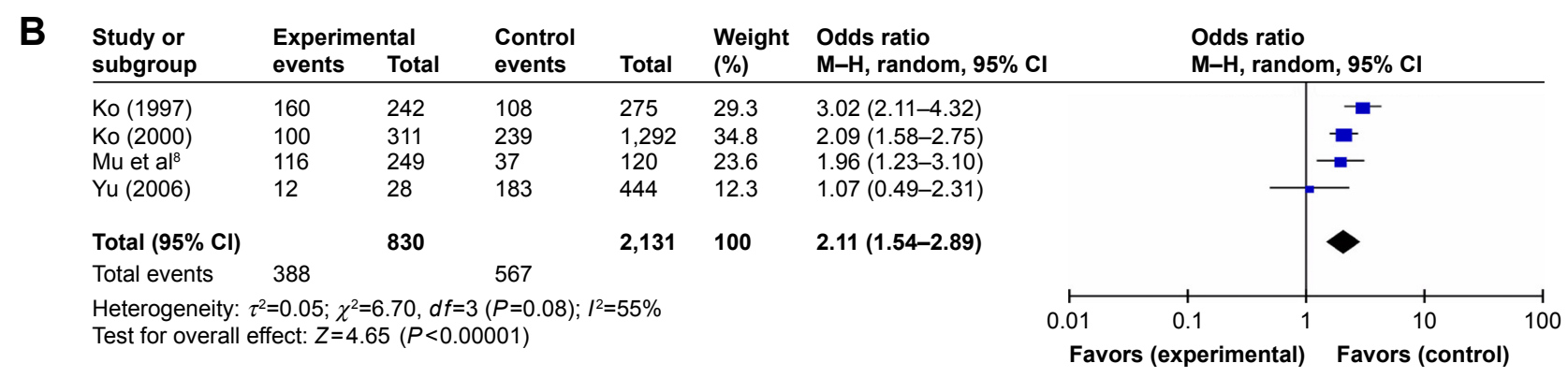

Figure 2 The forest plot of OR for lung cancer with studies included in this meta-analysis.

Notes: (A) Forest plots of meta-analysis for association between cooking oil fume exposure and lung cancer among Chinese nonsmoking women. (B) Correlation of lacking a kitchen ventilator with risk of lung cancer among Chinese nonsmoking women exposed to cooking oil fume. The total RR and $95 \% \mathrm{Cl}$ are summarized with fixed effects model or random effects model.

Abbreviations: $\mathrm{Cl}$, confidence interval; $d f$, degrees of freedom; $\mathrm{RR}$, risk ratio; OR, odds ratio. 
A

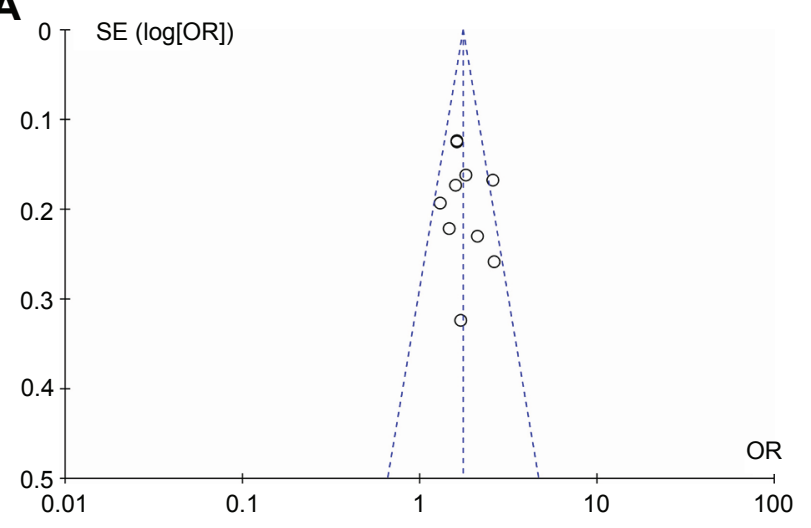

B

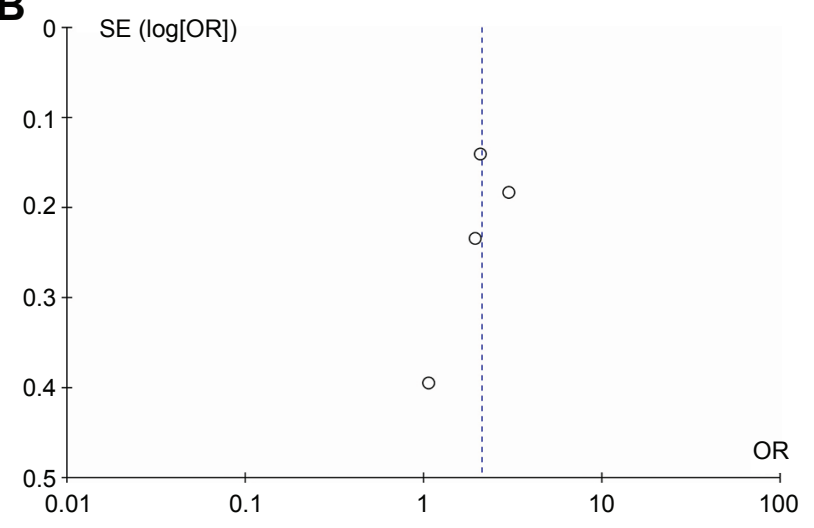

Figure 3 Funnel plots for the meta-analysis suggest that there was no obvious publication bias.

Notes: (A) Funnel plots for association between cooking oil fume exposure and lung cancer among Chinese nonsmoking women. (B) Funnel plots for correlation of lacking a kitchen ventilator with risk of lung cancer among Chinese nonsmoking women exposed to cooking oil fume.

Abbreviations: SE, standard error; OR, odds ratio.

3-(4,5-dimethyl-2-thiazolyl)- 2,5-diphenyl-2-H-tetrazolium bromide (MTT) cell proliferation assay showed that A549, a lung adenocarcinoma cell line, with cooking oil fume exposure for 48 hours exhibited significantly increased cell viability compared with control group. Nuclear factor kappa B facilitates the expression of inhibitor of apoptosis 2 (IAP2), an inhibitor of apoptosis proteins, to fully inhibit the tumor necrosis factorinduced apoptosis under cooking oil fumes and 2,4-decadienal condition. ${ }^{24}$ To date, quite a number of biochemistry studies in vivo have revealed the mechanism by which cooking oil fumes improve lung adenocarcinoma cell survival.

In the past few years, although a large number of studies on nosetiology of lung cancer are performed, the results are inconclusive. Cigarette smoking is thought to be an impact factor of lung cancer, but the epidemiologic characteristics of lung cancer in women who smoke rarely or never smoke remain complicated..$^{25}$ Genetic and environmental factors, especially occupational exposure, may also increase the risk of lung cancer. Indoor particulate matter level and various indoor poisonous air accelerate the incidence rate of lung cancer among lifetime never smokers in Taiyuan, People's Republic of China. ${ }^{8}$ Yin et $\mathrm{a}^{26}$ found that the cooking oil fume exposure was associated with high risks of lung cancer among nonsmoking females in the People's Republic of China, and telomerase reverse transcriptase (TERT) polymorphism (rs2736100) might be a genetic susceptibility factor. Although numerous studies have investigated the association between cooking oil fume and risk of lung cancer in nonsmoking women, the results remain uncertain at present.

To our knowledge, we for the first time conducted a metaanalysis to summarize the association between oil fuel smoke and lung cancer in Chinese nonsmoking women. The results showed that cooking oil fume exposure significantly increases the risk of lung cancer in nonsmoking female population. However, some limitations do exist in this meta-analysis. First, although we did our best to obtain all the publications, some studies may not have been included in this analysis due to lack of detailed data; thus, publication bias may exist in this meta-analysis. Second, other factors in the studies may increase between-study heterogeneity. The heterogeneity may be from regional differences, indoor PM2.5, coal type, outdoor air quality, housing characteristics, second-hand smoking, and previous lung disease. Third, most of the study locations were in the People's Republic of China, which may increase the heterogeneity and result in publication bias. Therefore, further high-quality studies in rural or urban regions of different countries with a high incidence of lung cancer still should be conducted in future to elucidate the causes of lung cancer.

\section{Conclusion}

In summary, this meta-analysis sheds light on the relationship between cooking oil fume exposure and lung cancer in Chinese nonsmoking female population. Our results show that long-term cooking oil fume exposure significantly increases the risk of lung cancer among Chinese nonsmoking women. The use of a kitchen smoke exhaust ventilator when cooking may reduce the risk of lung cancer among nonsmoking women. Therefore, some improvable strategies in cooking habits, gas filtration, and kitchen ventilation should be used to attenuate the harmful effects of cooking oil fume on human health. A meta-analysis including more high-quality epidemiological studies and passive smoking stratified analysis is necessary in the future.

\section{Disclosure}

The authors report no conflicts of interest in this work. 


\section{References}

1. Liu X, Lin X, Wang C, et al. Association between smoking and p53 mutation in lung cancer: a meta-analysis. Clin Oncol. 2014;269(1):18-24.

2. Parkin D, Whelan S, Ferlay J, Teppo L, Thomas D. Cancer Incidence in Five Continents. Vol. VIII. Lyon: IARC Scientific Publications; 2002:155.

3. Clément-Duchêne C, Wakelee H. Lung cancer incidence in never smokers. Eur J Clin Med Oncol. 2010;2(2):49-57.

4. Sun S, Schiller JH, Gazdar AF. Lung cancer in never smokers-a different disease. Nat Rev Cancer. 2007;7(10):778-790.

5. Turner MC, Krewski D, Pope CA 3rd, Chen Y, Gapstur SM, Thun MJ. Long-term ambient fine particulate matter air pollution and lung cancer in a large cohort of never-smokers. Am J Respir Crit Care Med. 2011; 184(12):1374-1381.

6. Mengersen K, Morawska L, Wang H, et al. The effect of housing characteristics and occupant activities on the respiratory health of women and children in Lao PDR. Sci Total Environ. 2011;409(8):1378-1384.

7. Wang A, Kubo J, Luo J, et al. Active and passive smoking in relation to lung cancer incidence in the Women's Health Initiative Observational Study prospective cohort. Ann Oncol. 2015;26(1):221-230.

8. Mu L, Liu L, Niu R, et al. Indoor air pollution and risk of lung cancer among Chinese female non-smokers. Cancer Causes Control. 2013; 24(3):439-450.

9. Yin Z, Cui Z, Guan P, et al. Interaction between polymorphisms in Pre-MiRNA genes and cooking oil fume exposure on the risk of lung cancer in Chinese non-smoking female population. PLoS One. 2015;10(6):e0128572.

10. Denholm R, Schüz J, Straif K, et al. Is previous respiratory disease a risk factor for lung cancer? Am J Respir Crit Care Med. 2014;190(5): 549-559.

11. Turati F, Rossi M, Pelucchi C, Levi F, La Vecchia C. Fruit and vegetables and cancer risk: a review of southern European studies. Br J Nutr. 2015;113(Suppl 2):S102-S110.

12. Taioli E, Zocchetti C, Garte S. Models of interaction between metabolic genes and environmental exposure in cancer susceptibility. Environ Health Perspect. 1998;106(2):67-70.

13. Du B, Wang Z, Zhang X, et al. MicroRNA-545 suppresses cell proliferation by targeting cyclin D1 and CDK4 in lung cancer cells. PLoS One. 2014;9(2):e88022.

14. Lai CH, Jaakkola JJ, Chuang CY, et al. Exposure to cooking oil fumes and oxidative damages: a longitudinal study in Chinese military cooks. J Expo Sci Environ Epidemiol. 2013;23(1):94-100.

15. Liu Y, Chen YY, Cao JY, et al. Oxidative stress, apoptosis, and cell cycle arrest are induced in primary fetal alveolar type II epithelial cells exposed to fine particulate matter from cooking oil fumes. Environ $\mathrm{Sci}$ Pollut Res Int. 2015;22(13):9728-9741.

16. Chiang TA, Wu PF, Ko YC. Identification of carcinogens in cooking oil fumes. Environ Res. 1999;81(1):18-22.

17. Wu SC, Yen GC. Effects of cooking oil fumes on the genotoxicity and oxidative stress in human lung carcinoma (A-549) cells. Toxicol In Vitro. 2004;18(5):571-580.

18. Yu IT, Chiu Y, Au JS, Wong TW, Tang J. Dose-response relationship between cooking fumes exposures and lung cancer among Chinese nonsmoking women. Cancer Res. 2006;66(9):4961-4967.

19. Lee T, Gany F. Cooking oil fumes and lung cancer: a review of the literature in the context of the US population. J Immigr Minor Health. 2013;15(3):646-652.

OncoTargets and Therapy

\section{Publish your work in this journal}

OncoTargets and Therapy is an international, peer-reviewed, open access journal focusing on the pathological basis of all cancers, potential targets for therapy and treatment protocols employed to improve the management of cancer patients. The journal also focuses on the impact of management programs and new therapeutic agents and protocols on
20. Lan Q, Chapman RS, Schreinemachers DM, Tian L, He X. Household stove improvement and risk of lung cancer in Xuanwei, China. J Natl Cancer Inst. 2002;94(11):826-835.

21. Li M, Yin Z, Guan P, et al. XRCC1 polymorphisms, cooking oil fume and lung cancer in Chinese women nonsmokers. Lung Cancer. 2008;62(2):145-151.

22. Chang LW, Lo WS, Lin P. Trans, trans-2, 4-decadienal, a product found in cooking oil fumes, induces cell proliferation and cytokine production due to reactive oxygen species in human bronchial epithelial cells. Toxicol Sci. 2005;87(2):337-343.

23. Yang SC, Jenq SN, Kang ZC, Lee H. Identification of benzo [a] pyrene 7, 8-diol 9, 10-epoxide N2-deoxyguanosine in human lung adenocarcinoma cells exposed to cooking oil fumes from frying fish under domestic conditions. Chem Res Toxicol. 2000;13(10):1046-1050.

24. Hung HS, Wu WJ, Cheng YW, Wu MF, Chang KL, Lee H. Cooking oil fumes improve lung adenocarcinoma cell survival through c-IAP2 induction. J Toxicol Environ Health A. 2005;68(17-18):1525-1535.

25. Pesch B, Kendzia B, Gustavsson P, et al. Cigarette smoking and lung cancer-relative risk estimates for the major histological types from a pooled analysis of case-control studies. Int J Cancer. 2012;131(5): 1210-1219.

26. Yin Z, Cui Z, Ren Y, et al. Genetic polymorphisms of TERT and CLPTM1L, cooking oil fume exposure, and risk of lung cancer: a case-control study in a Chinese non-smoking female population. Med Oncol. 2014;31(8):1-6.

27. Ko YC, Lee CH, Chen MJ, et al. Risk factors for primary lung cancer among non-smoking women in Taiwan. Int J Epidemiol. 1997;26(1): 24-31.

28. Liu DK, Shen YP, Gao LF, Jin F. The case-control study of lung cancer risk factors in Shanghai. Chin Tumor. 2000;9(3):120-121.

29. Liu EJ, Xiang YB, Jin F, Gao YT. Risk factors for lung cancer among non-smoking females in urban Shanghai: a population-based casecontrol study. Tumor (Shanghai). 2001;21(6):421-425.

30. Metayer C, Wang ZY, Kleinerman RA, et al. Cooking oil fumes and risk of lung cancer in women of rural Gansu (in Chinese). Chin Pre Med. 2002;3(3):186-190.

31. Su M, Zhou BS. Association of genetic polymorphisms in $I L-6$ and $I L-1 \beta$ gene with risk of lung cancer in female non-smokers (in Chinese). Chin J Lung Cancer. 2014;17(8):612-617.

32. Wang T, Zhou B, Shi J. Lung cancer in nonsmoking Chinese women: a case-control study. Lung cancer. 1996;14:S93-S98.

33. Zhong L, Goldberg MS, Gao YT, et al. Lung cancer and indoor air pollution arising from Chinese-style cooking among nonsmoking women living in Shanghai, China. Epidemiology. 1999;10(5):488-494.

34. Zhu XX, Hu CB, Gu QH. CYPIAI polymorphisms, lack of glutathione S-transferase (GSTM1), cooking oil fumes and lung cancer risk in non-smoking women (in Chinese). Chin J Tuberc Respir Dis. 2010; 33(11):817-822.

35. Ko YC, Cheng LSC, Lee CH, et al. Chinese food cooking and lung cancer in women nonsmokers[J]. Am J Epidemiol. 2000;151(2):140-147.

36. Yu ITS, Chiu Y, Au JSK, et al. Dose-response relationship between cooking fumes exposures and lung cancer among Chinese nonsmoking women[J]. Cancer Res. 2006;66(9):4961-4967.

patient perspectives such as quality of life, adherence and satisfaction The manuscript management system is completely online and includes a very quick and fair peer-review system, which is all easy to use. Visit http://www.dovepress.com/testimonials.php to read real quotes from published authors. 